\title{
LA COMPETITIVIDAD: ¿ES UNA OBSESIÓN O UNA NECESIDAD?
}

\section{COMPETITIVENESS: IS IT AN OBSESSION OR A NEED?}

\section{JeAn P. SeClen Luna*}

[Recepción: Abril de 2009 / Conformidad: Mayo de 2009]

\section{RESUMEN}

"La competitividad despierta un interés floreciente en grupos variopintos: los políticos pretenden mejorarla, los legisladores debaten sobre ella, los editores publican sobre ella, los consultores viven de implantarla, y los economistas intentan explicarla y medirla”. Esta frase es, sin duda, la base para reflexionar y aclarar la importancia de la competitividad en la vida cotidiana. Claro está que detrás de esto subyace de manera sutil, pero no menos importante, la necesidad de comprender bien este concepto, ya que de una manera $u$ otra todos nos vemos inmersos en estos derroteros.

Palabras clave: Competitividad, ventajas competitivas y fuentes de ventajas competitivas.

\begin{abstract}
"Competitiveness is of growing interest in diverse groups: politicians try to improve it, lawmakers debate over it, editors publish on it, consultants make a living out of implanting it, and economists try to explain it and measure it." This phrase is undoubtedly the basis to think about and to clarify the importance of competitiveness in daily life. It is clear that behind it lies a subtle but not less important need to understand this concept, because in one way or another we are all involved in these courses.
\end{abstract}

Keywords: Competitiveness, Competitive Advantage, Source of Competitive Advantage.

* Doctorando en Economía de la Empresa. Facultad de Ciencias Económicas y Empresariales de la Universidad del País Vasco. Enero de 2009. 


\section{INTRODUCCIÓN}

En muchas ocasiones, la competitividad parece el nuevo nirvana donde se solucionan todos los problemas. Corrientes económicas liberales e intervencionistas acuden a ella como reto y mito con el que justificar sus propias posiciones. En aras de la competitividad se puede defender tanto el mayor de los proteccionismos (aduciendo el interés nacional, la seguridad de los trabajadores, etc.) como el más alto liberalismo (en función de la total desregulación y liberalización con la que las empresas reducirán costes y podrían competir mejor). Para Krugman (1996), el miedo a perder posiciones en el comercio internacional es infundado y puede considerarse un mito. Que la productividad de un país crezca por debajo de la de sus socios comerciales no constituye un factor de pérdida ni de comercio ni de bienestar. La competitividad es un concepto crecientemente popular. Así, la expresión competitividad se circunscribía inicialmente a la economía empresarial, y la capacidad de una empresa para ser competitiva se valoraba en relación con sus posibilidades de mantener o aumentar la rentabilidad de sus activos en las condiciones prevalecientes en mercados abiertos a la competencia. El hecho de que la ganancia de cuota de mercado, por parte de una empresa, deba tener lugar a costa de las demás empresas que operan en el mismo sector vincula la idea de competitividad empresarial con la de rivalidad.

La aplicación del concepto de competitividad no ya a empresas concretas, sino a regiones o países enteros, representa un cambio de escala que presenta importantes riesgos desde el punto de vista analítico. En primer lugar, a escala nacional o regional no existe un equivalente a la quiebra empresarial. En segundo lugar, los números rojos en la balanza comercial de un país o de una re- gión no son el equivalente de las pérdidas registradas por la contabilidad de una empresa. En vez de constituir un síntoma indiscutible de fracaso competitivo constituyen más bien un indicador de la existencia temporal de un desequilibrio macroeconómico entre gasto y producción interna. Igualmente, la ganancia de peso relativo de las exportaciones de un país en el mercado internacional no posee automáticamente un significado inequívoco en términos de mejora de bienestar nacional, ya que dependerá de las causas que la hayan originado. No es lo mismo si responde a la capacidad de sus empresas para ofrecer productos altamente diferenciados que gocen del favor de los consumidores de otros países que si es el fruto temporal de una recesión en el mercado interno, acompañada de una depreciación de la divisa nacional. Nuevamente, Krugman considera como una 'obsesión peligrosa' la tendencia a considerar las relaciones económicas internacionales bajo el prisma de la rivalidad competitiva. Con ello, los países no compiten entre sí del mismo modo que las empresas, ya que la interdependencia entre ellos es mucho más rica y variada: el mutuo interés por acceder a sus mercados respectivos es un ejemplo de ello.

La historia económica de la segunda mitad del siglo XX avala en líneas generales la tesis de la prosperidad compartida a través del comercio, al coincidir la decadencia de proteccionismo comercial con un periodo de intenso crecimiento económico. La constatación de los riesgos implícitos en trasladar mecánicamente la visión de la competitividad propia del mundo empresarial a unidades territoriales como países o regiones, ha llevado a la búsqueda de variables que de un lado pudieran asociarse a la idea de competitividad y de otro tuvieran una significación inequívoca en términos de bienestar. De este modo, la atención se ha desplazado ha- 
cia aspectos relacionados con el crecimiento económico y, en particular, hacia una de sus variables clave: la productividad del trabajo. Las mejoras de la productividad del trabajo no sólo contribuyen al bienestar de la población a través de la mejora de los salarios reales o de la reducción potencial de la jornada laboral, sino que crean recursos disponibles para la inversión y ofrecen a largo plazo una base fiscal sólida para la ampliación de la oferta de bienes públicos.

\section{2. ¿QUÉ SIGNIFICA COMPETITIVIDAD?}

La posibilidad de crecimiento, generación de valor y capacidad de competir de las empresas estuvo fundada durante años en el análisis del entorno competitivo (aprovechamiento de las imperfecciones del mercado), con un importante sustento de las cinco fuerzas competitivas planteadas por Porter (1987); pero la nueva concepción de la empresa como un conjunto de recursos y capacidades heterogéneas que pueden utilizarse para crear una posición exclusiva en el mercado, dio paso a la denominada visión de la firma basada en los recursos (Wernerfelt, 1984). Así, es abundante la literatura que afirma repetidamente que la competitividad es un concepto mal definido, esto se debe a la falta de claridad en la concreción de la unidad a que nos referimos: ¿hablamos de competitividad nacional, de competitividad de una industria o competitividad de una empresa? Existen diferentes líneas de investigación y teorías de carácter económico que han tratado de identificar el origen de la competitividad; sin embargo, es posible apreciar cierto consenso entre los investigadores al señalar que la competitividad viene condicionada o determinada por tres tipos de factores o fuen- tes: los relativos al país donde la empresa se ubica, "efecto país"; los derivados del sector al que pertenece, "efecto sector" y los que tienen su origen en la propia empresa, "efecto empresa" (Cuervo, 1983; Salas, 1983; Galán y Vecino, 1997). Las tres dimensiones son significativas, puesto que permiten a la empresa encontrar ventajas competitivas.

Cuando se refiere a la competitividad internacional de un país, la nebulosa es cierta (Dollard y Wolff, 1993; Krugman, 1994; Waheeduzzaman y Riaño, 1996). Pese a que la teoría económica clásica ha reducido el trabajo a la comparación de magnitudes como precios, tipos de cambio o flujos exteriores, el efecto país recoge aquellos factores del entorno macroeconómico, político, administrativo y cultural que favorecen o entorpecen la competitividad de las empresas ubicadas en un determinado territorio frente a las empresas que no se ubican en ese territorio. Es decir, se trata de factores como el sistema de ciencia y tecnología existente, la concentración de servicios avanzados a empresas, las políticas desarrolladas por la Administración Pública, la flexibilidad del mercado laboral, la disponibilidad de infraestructuras, el grado de autonomía energética, etc.

En cambio, cuando la competitividad se refiere al sector, existe mayor consenso (Mason, 1939; Bain, 1956; Caves y Porter, 1977; Scherer, 1980; Scherer y Ross, 1990; Porter, 1981, 1982; Dollard y Wolff, 1993; Puig, 1996). En definitiva, el efecto sector señala la estructura del sector industrial al que pertenece la empresa como el principal determinante de su rentabilidad. Por tanto, la estructura del sector (grado de concentración, diferenciación del producto, barreras de entrada para competidores potenciales, tasa de crecimiento, poder de negociación de clientes y proveedores, etc.) van a determinar el grado de intensidad y rivalidad competitiva y 
la rentabilidad de las empresas pertenecientes al mismo sector.

Por último, cuando la competitividad se refiere a una empresa, también existe un mayor consenso (Penrose, 1959; Andrews, 1971; Wernerfelt, 1984; Teece, 1986; Prahalad y Hamel, 1990; Barney, 1991; Grant, 1991; Amit y Schoemaker, 1993; Peteraf, 1993; Gautman et áal, 2004). El efecto empresa se basa en el hecho de que en un mismo sector existen empresas más o menos competitivas y ello se explica por factores internos de cada empresa y no por cuestiones del sector. Es decir, son los recursos y capacidades de cada empresa los que les permiten distinguirse de las demás, y gestionados adecuadamente permitirán obtener ventajas competitivas que harán posible que empresas de una misma industria obtengan niveles de rentabilidad diferentes.

Como vemos, son muchas las investigaciones que centran su atención en la determinación de los distintos efectos (país, sector, empresa) y en el estudio de la importancia relativa que cada uno de ellos tiene en la determinación del éxito competitivo de las empresas. Sin embargo, no se puede afirmar concluyentemente cual es la importancia relativa en el éxito empresarial de cada una de estas tres fuentes de competitividad. No obstante, Claver et áal (2002) realizan una revisión de los trabajos previos tratando de analizar la importancia relativa, y concluyen que la mayor parte de las investigaciones previas más recientes indican que el efecto empresa tiene una mayor importancia relativa que el efecto sector.

\section{DEFINICIÓN DEL CONCEPTO DE COMPETITIVIDAD Y VENTAJA COMPETITIVA}

Para algunos autores, el término competitividad procede de competencia. En el ám- bito económico, este término tiene dos significados: el grado de rivalidad económica existente en un mercado, o bien, la forma de actuación de los agentes en el mismo. Se trata también de un concepto relativo, "se es competitivo si se está en condiciones de competir con otro" (Viedma, 1992; Cuervo, 1993; Alonso y Barcenilla, 1999). Es decir, siempre está en función de una comparación exigente con las empresas rivales, habitualmente referidas a la posición y futuro de las empresas en el mercado. No obstante, tiene un significado algo difuso y difícil de calibrar correctamente en toda su extensión, puesto que también la competitividad de una empresa tiene que ver, necesariamente, con su capacidad para adaptarse en el tiempo, entendida como respuesta a la evolución natural paralela al marco socioeconómico y tecnológico.

Camisón (1997) considera los conceptos de competitividad empresarial y éxito empresarial como equivalentes y propone unificar ambos en el concepto de éxito competitivo, así, afirma que competitividad significa literalmente "capacidad para competir". Por tanto, es un concepto cuya utilidad se limita a entes (unidades económicas, sectores o economías) que actúan dentro de un sistema de competencia para lograr ciertos objetivos. Así, el problema de ¿cómo ser competitivos? es vital para la supervivencia, al constituir una referencia de la capacidad de anticipación y de respuesta de la empresa ante retos del entorno. Así, el éxito competitivo se manifiesta por "la capacidad de una empresa para, a través de una gestión eficiente de una cierta combinación de recursos disponibles y del desarrollo de determinadas estrategias, configurar una oferta de productos que resulten atractivas para una cuota significativa de mercado, y le permitan, desarrollar una ventaja competitiva que sea sostenible en el tiempo y le haga capaz de alcanzar una po- 
sición competitiva favorable, por mantener y aumentar su participación en el mercado al tiempo que logra unos resultados financieros superiores y un crecimiento satisfactorio de las rentas generadas, sin necesidad de recurrir a una remuneración anormalmente baja de los factores de producción".

En definitiva, la definición de competitividad obedece claramente a una orientación hacia el mercado y podemos entenderla como una medida del éxito de una organización para sobrevivir con futuro en un entorno dinámico. El resultado por alcanzar será mayor dependiendo del adecuado posicionamiento que pueda obtener la empresa en el mercado con respecto a los competidores para la satisfacción de la demanda presente y futura de los clientes. Es decir, la competitividad es la capacidad para generar ventajas competitivas sostenibles, que permitan producir bienes y servicios con valor para los compradores, a través de factores o recursos esenciales como consecuencia de la rivalidad suscitada en relación con otras empresas.

La ventaja competitiva hace entonces referencia a la posesión de la empresa por ciertos elementos (recursos, capacidades, productos, etc.) en una medida en la cual carecen sus competidores, que le permite obtener una posición competitiva superior.

La posición competitiva hace referencia al lugar que ocupa la empresa en relación con su competencia dentro de cierto negocio, sobre la base de un cierto número de factores o variables de medida. Por consiguiente, el concepto de competitividad adoptado deja claro que son esencialmente las empresas las organizaciones que compiten en el mercado, siendo pues el ámbito empresarial el más acertado como base para el análisis de los factores determinantes de la competitividad. En cualquier caso, como hemos mencionado, la forma de crear y sostener las ventajas competitivas tiene un origen que puede ser múltiple.

\section{FUENTES DE VENTAJAS COMPETITIVAS}

Se ha identificado cuatro fuentes principales: las competencias distintivas, la cadena de valor, la calidad total, y la innovación. (Figura 1).

Las competencias distintivas. En el trabajo clásico de Selznick (1957), se acuñaba ya el concepto de competencia distintiva de una organización, no como lo que puede hacer, sino como lo que puede hacerse bien. Andrews (1971) le da ya una concepción actual al referirla a la experiencia en reproducir y comercializar una línea de productos, desarrollo de las capacidades de los individuos que comprende la organización, el grado en que la capacidad individual se aplica a la tarea común, y la calidad de la coordinación del esfuerzo individual y de grupo. La orientación predominante en la Dirección Estratégica ha asumido ya el papel decisivo de la heterogeneidad de cada empresa (su competencia distintiva) como fuentes de ventajas competitivas, de modo que tanto el análisis estratégico como en la fase de implantación de la estrategia, adquieren un relieve prioritario elementos tales como: la cultura de empresa, el mapa de poderes y el juego político existente en la organización, la peculiaridad de su arquitectura organizativa o las destrezas diferenciadas de sus recursos humanos. Así, el concepto "competencias distintivas" ha contado con la cooperación de tres líneas de investigación de los orígenes de las ventajas competitivas intrínsecas a la empresa: la Teoría de la Excelencia Organizativa, la Teoría de la Estrategia basada en las Competencias y la Teoría de Recursos y Capacidades. 
FIGURA 1: FUENTES DE VENTAJAS COMPETITIVAS

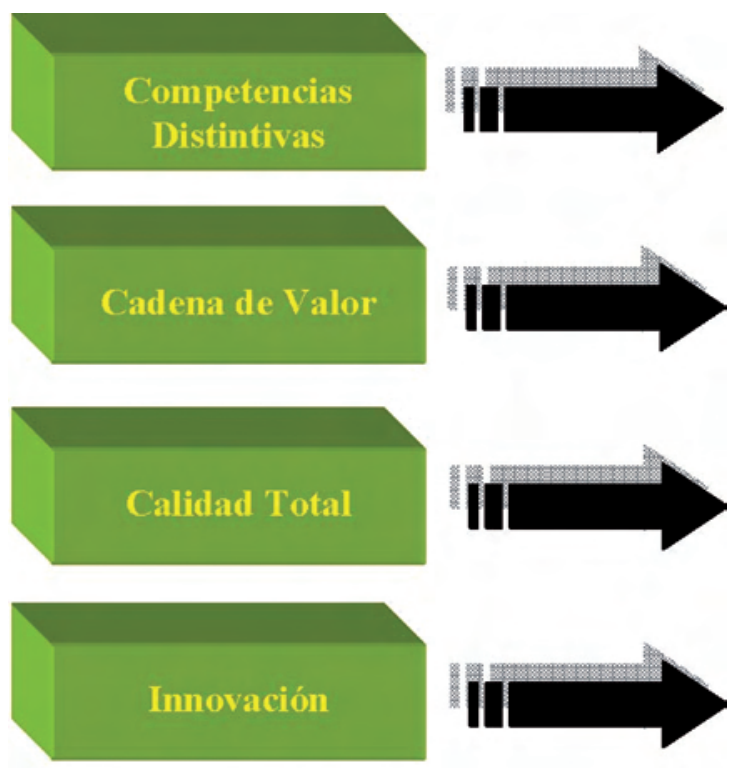

Habilidades que requieren dominio conjunto de tecnologías convencionales y traducen el aprendizaje colectivo de una organización. "Aquello que se sabe hacer bien de verdad".

Conjunto de tareas diferenciadas y enlazadas, cada una de las cuales va contribuyendo sucesiva y proporcionalmente a la formación del valor del producto o servicio.

Su gestión permite crear sinergias en todas las actividades de la empresa, desde las personas hasta los procesos, clientes, proveedores y competidores.

El proceso de innovación es capaz de generar rentas, es difícil de imitar y es acumulativo. Además, su gestión integral permite reforzar la sostenibilidad de las ventajas competitivas.

Fuente: Elaboración propia.

La Teoría de la Excelencia Organizativa tuvo su primera aportación con el famoso "Esquema McKinsey 7-S" de Peters y Watermann (1982), que intentaban explicar la competitividad de una organización a partir de 7 variables: dos de ellas acerca del "hardware" de la organización (la estructura y la estrategia), y otras cinco acerca del "software" de la organización (estilo, sistemas, personal, destrezas y valores compartidos). Sobre estos siete bloques de complejidad, que explicaría el proceso de cambio en la empresa, abordaron la identificación de los atributos que permiten distinguir las empresas sobresalientes. Estos atributos se deberían enfocar en dos áreas claves: el desempeño financiero a largo plazo y la capacidad de innovación. Quizás, las dos críticas más profundas son las que hacen referencia a la construcción de una medida que permita operativizar los siete atributos del modelo y a la relación entre estrategia y estructura.

La Teoría de la Estrategia basada en las Competencias está estrechamente vinculada con el concepto de "competencias nucleares" o "core competences" (Prahalad y Hamel, 1990) y el énfasis sobre los activos intangibles como raíces esenciales de las ventajas competitivas. Las competencias distintivas son la principal fortaleza de la organización, y deben consecuentemente guiar la elección de la estrategia. Una estrategia competitiva sólida debe basarse en la conjunción singular de una oportunidad percibida en el entorno. Esta idea rompe abiertamente con los enfoques cuantitativos predominantes en la Teoría de la Estrategia de los años 80, principalmente con los modelos de carteras, cuyos conceptos centrales (economías de escala, curva de aprendizaje y cuota de mercado) estaban relacionadas con la obsesión por los costes y el tamaño. Así, la incapacidad de empresas grandes, establecidas como líderes del negocio, para defenderse de empresas más pequeñas no podía estribar en la disponibilidad de los recursos tradicionales.

Las respuestas tradicionales de corte estático, basadas en el análisis de estructuras de costes o de factores de la industria o del en- 
torno en general, eran incapaces de aportar pista alguna sobre por qué ciertas empresas eran más competitivas con menos medios. Así, la teoría de Hamel y Prahalad fue que la causa esencial era la posesión de una visión de futuro a largo plazo que guiaba a todos sus actos, muy distintos a sus objetivos financieros a corto plazo. Por tanto, estos autores conceden a la identificación de las competencias esenciales de una empresa, una noción que va más allá de "puntos fuertes" de la empresa. Es decir, dichas competencias son difícilmente imitables por los competidores, y ofrecen por tanto el más amplio potencial para crear ventajas competitivas sostenibles sobre la base de su plasmación en características de diferenciación del producto que el consumidor perciba como especial.

Finalmente, la Teoría de Recursos y Capacidades frente a los postulados de la Economía Industrial que defienden que las diferencias en las rentabilidades dependen de la estructura competitiva del sector en que se ubica la empresa, aporta evidencias de la importancia de elementos internos de la empresa para encontrar las verdaderas justificaciones de las diferencias de resultados, convirtiendo a la empresa en una unidad de análisis. En otras palabras, los modelos que se centran en el análisis atractivo del sector, donde la ventaja competitiva sostenible viene vía posición de los mercados de productos, presuponen dos hipótesis de partida subyacentes: (a) los recursos que manejan las empresas de un mismo sector y las estrategias que llevan a cabo son homogéneos; (b) en el caso que exista heterogeneidad de recursos, ésta tendrá una corta duración puesto que los recursos son altamente móviles (existe un mercado de factores donde tendrá lugar su compra-venta). En la Teoría de Recursos, sin embargo, es un planteamiento donde el énfasis se pone en el interior de la empresa y en su relación con la estrategia - resultados, esos dos supuestos se rechazan y se trabajan con los dos siguientes: (a) los recursos que manejan las empresas de un determinado sector pueden ser idiosincrásicos, y por tanto, las empresas podrán ser heterogéneas; (b) dicha heterogeneidad puede ser de larga duración puesto que los recursos que pueden controlar las empresas no serán perfectamente móviles. En gran medida porque sus mercados o son muy imperfectos o no existen. Esta característica asegura la permanencia de la heterogeneidad en el tiempo y propicia la obtención de rentas ricardianas.

Resumiendo, bajo estos planteamientos se puede empezar a vislumbrar cómo la competitividad de la empresa dependerá de la dotación de recursos que disponga, así como de su capacidad interna para coordinarlas (Fernández, 1993). Además, para que los recursos y capacidades de la empresa actúen como competencias distintivas deben cumplir una serie de características (Amit y Schoemaker, 1993): ser duraderos (sufran menos desgaste posible) y escasos (exclusivos), inimitables (que no se puedan replicar), de sustitución limitada (no tenga problemas de duplicación o de obsolescencia), no comercializables (inmóviles), complementarios entre sí en el proceso de generación de valor, de transferencia difícil (ambigüedad causal), finalmente, deben solaparse con los factores estratégicos del sector. (Cuadro 2).

La cadena de valor. Ésta es una técnica de análisis estratégico que fue desarrollada por Porter (1985), ya que suele tener una gran aplicación en cualquier empresa independientemente de cuál sea su tamaño o sector de actividad. La cadena de valor es una herramienta que sirve de ayuda para determinar los fundamentos de la ventaja competitiva al centrarse en el valor a transmitir desde la em- 
CUADRO 2: REQUISITOS DE LOS RECURSOS ESTRATÉGICOS

\begin{tabular}{|c|c|c|c|c|c|c|}
\hline $\begin{array}{c}\text { Dierickx y Cool } \\
\text { (1989) }\end{array}$ & $\begin{array}{c}\text { Reed y De } \\
\text { Fillippi } \\
(1990)\end{array}$ & $\begin{array}{l}\text { Grant } \\
\text { (1991) }\end{array}$ & $\begin{array}{l}\text { Barney } \\
(1991)\end{array}$ & $\begin{array}{l}\text { Peteraf } \\
(1993)\end{array}$ & $\begin{array}{l}\text { Strategor } \\
\text { (1993) }\end{array}$ & $\begin{array}{c}\text { Amit y } \\
\text { Schoemaker } \\
(1993)\end{array}$ \\
\hline \multirow[t]{3}{*}{$\begin{array}{l}\text { Interdependencia } \\
\text { de los activos }\end{array}$} & $\begin{array}{l}\text { Ambigüedad } \\
\text { Causal: } \\
\text { Carácter tácito } \\
\text { Complejidad } \\
\text { Especificidad }\end{array}$ & Durabilidad & $\begin{array}{l}\text { Valiosos y } \\
\text { escasos }\end{array}$ & Heterogeneidad & Raros & $\begin{array}{l}\text { Durabilidad y } \\
\text { escasez }\end{array}$ \\
\hline & & $\begin{array}{l}\text { Replicabilidad } \\
\text { imperfecta }\end{array}$ & No sustituibles & No sustituible & No sustituible & No sustituible \\
\hline & & $\begin{array}{l}\text { Transferencia } \\
\text { imperfecta: } \\
\text { Inmovilidad } \\
\text { Información } \\
\text { imperfecta } \\
\text { Especificidad } \\
\text { Apropiabilidad }\end{array}$ & $\begin{array}{l}\text { No imitables: } \\
\text { Dependencia } \\
\text { de la historia } \\
\text { Ambigüedad } \\
\text { causal } \\
\text { Complejidad } \\
\text { social }\end{array}$ & $\begin{array}{l}\text { No imitable } \\
\text { Imperfecta } \\
\text { movilidad } \\
\text { Rentas } \\
\text { compartidas }\end{array}$ & $\begin{array}{l}\text { No imitable: } \\
\text { Ambigüedad } \\
\text { causal } \\
\text { Complejidad } \\
\text { Confidencialidad } \\
\text { Difícilmente } \\
\text { accesibles }\end{array}$ & $\begin{array}{c}\text { No imitable } \\
\text { No comercializable } \\
\text { Complementarios } \\
\text { Apropiabilidad } \\
\text { Solapamiento } \\
\text { con factores } \\
\text { estratégicos de la } \\
\text { industria }\end{array}$ \\
\hline
\end{tabular}

Fuente: Elaboración propia a partir de Fernández y Suárez (1996).

presa a los clientes. Bajo el razonamiento que entiende que la búsqueda y mantenimiento de las ventajas competitivas no pueden ser comprendidas mirando a la empresa como un todo, Porter utiliza el concepto de "cadena de valor" y lo aplica tanto para un sector como para cualquiera de las empresas que lo forman. A pesar que Porter popularizó este concepto, originalmente la empresa de consultoría estratégica Mc Kinsey \& Co. desarrolló una visión inicial denominada "sistema de negocio" (como ya hemos detallado anteriormente), que distinguía una serie de funciones básicas a las que les señala unos temas relevantes. Así, el enfoque funcional comprendía las siguientes áreas: tecnología (fuente, sofistificación, patentes, etc.), diseño del producto (características, estética, calidad, etc.), producción (integración, capacidad, localización, ensamblado, etc.), márketing (precios, publicidad, promoción, marca, etc.), distribución (almacenes, canales, transporte, integración, etc.), servicio (naturaleza, garantía, rapidez, etc.).
El concepto de cadena de valor consiste en dividir la actividad de la empresa en un conjunto de tareas diferenciadas, denominadas actividades de producción de valor. Por tanto, su idea parte de contemplar a la empresa como un conjunto de actividades enlazadas, cada una de las cuales va contribuyendo sucesiva y proporcionalmente a la formación del valor total del producto, que se pretende recuperar con su venta en el mercado. En definitiva, éste es el valor que después apreciará el consumidor, bien por el coste final del producto o por la diferenciación que haya alcanzado la empresa frente a su competencia. Cada una de las fases de la cadena de valor y las tareas que implican, así como sus interrelaciones, pueden ser fuentes de ventajas competitivas. Para Porter, es una herramienta de análisis de las diferentes actividades que realiza la empresa, que permite una comprensión eficaz de las fuentes generadoras de ventajas competitivas. Por tanto, la meta de este análisis es la identificación de esas fuentes generadoras de ventajas. 
La calidad total. Aunque inicialmente el enfoque era hacia la calidad del producto, después fue hacia los clientes. Posteriormente, se dirige hacia todos los grupos de interés de la organización: clientes, accionistas, personas de la plantilla, proveedores y sociedad en general. La calidad total se pretende alcanzar mediante los "Modelos de Excelencia”, así, en Japón se pretende conseguir mediante el Modelo Deming, en Estados Unidos de Norteamérica mediante el Modelo Malcom Baldrige, en Europa el Modelo EFQM (European Foundation Quality Management), y en Iberoamérica mediante el FUNDIBEQ (Fundación Iberoamericana para la Gestión de la Calidad). En el caso que nos ocupa, en Europa la EFQM sirve para medir en qué grado las empresas aplican los principios de excelencia: enfocarse en el cliente, enfocarse en las personas, realizar la gestión por procesos, buscan la mejora y el aprendizaje continuo, la importancia del liderazgo, una clara orientación hacia los resultados, gestión de las alianzas y la responsabilidad social. No obstante, se suele incurrir en el error de querer implantar el modelo en la gestión diaria de la empresa, cuando en el fondo se trata de una guía de mejora. Por otra parte, la calidad total es necesaria pero no suficiente, porque requiere de tiempo y de priorizar las actividades dentro de una empresa para su implantación. Además, su principal handicap es que tiende a estandarizar la operatividad de las empresas y sobre todo a enraizar la inercia estructural de las mismas. Sin embargo, el Modelo EFQM y la gestión por procesos aportan las ideas básicas para la innovación.

La innovación. Se considera como el cambio en los productos y procesos, la mejora de los mismos, los nuevos enfoques del márketing o las nuevas formas de distribución (North et al, 2001). Estas nuevas ideas pueden mejorar la forma en que hasta el momento se están haciendo las cosas, o bien, cambiarlas radicalmente. La innovación puede producirse gracias a la investigación de la propia empresa o bien adquiriendo nuevas tecnologías o licencias. Pese a que las grandes empresas por poseer más medios financieros y humanos, pueden parecer más propensas a realizar innovaciones, sin embargo, las PYME también innovan. Sobre la base de la literatura, tres son las claves sobre las que se asienta la innovación en las PYME: el directivo, los trabajadores y la cooperación con otras empresas. Los recursos humanos son un elemento fundamental para el desarrollo de innovaciones muy especialmente en las empresas de menor tamaño. Los mayores niveles de comunicación existentes, los menores niveles de formalización, que dotan de una mayor autonomía a los trabajadores, y el mayor compromiso que se establece entre trabajador y empresa facilitan la creatividad de los trabajadores y su participación en el desarrollo de innovaciones. Es relevante que la adecuada gestión y sistemas de protección estén alineados con la estrategia corporativa de las empresas. Así, la innovación es una fuente de ventaja competitiva ya que a través de su proceso puede ser tácito, acumulativo y difícil de imitar. Es más, es el que mejor cumple con las características de las competencias distintivas antes descritas por Amit y Schoemaker (1993).

\section{CONCLUSIONES}

La competitividad viene condicionada por tres tipos de factores: los relativos al país donde la empresa se ubica "efecto país", los derivados del sector al que pertenece "efecto sector" y los que tienen su origen en la propia empresa "efecto empresa". Sin embargo, 
la mayor parte de las investigaciones indican que el efecto empresa tiene una mayor importancia relativa que los demás efectos.

La competitividad es la capacidad para generar ventajas competitivas sostenibles que permitan producir bienes y servicios con valor para los compradores, a través de factores o recursos esenciales como consecuencia de la rivalidad suscitada en relación con otras empresas.

Teniendo unos recursos y desarrollando unas capacidades a través de una "rutina" o aprendizaje, podemos conseguir una ventaja competitiva sostenible. Es decir, los recursos y las capacidades son el núcleo de las ventajas competitivas.

Existen cuatro principales fuentes de ventajas competitivas: las competencias distintivas (es aquello que se sabe hacer bien de verdad), la cadena de valor (conjunto de tareas diferenciadas y enlazadas, que va contribuyendo sucesiva y proporcionalmente a la formación del valor del producto o servicio), la calidad total (permite crear sinergias en todas las actividades de la empresa, desde las personas hasta los clientes, proveedores y competidores), y la innovación (es difícil de imitar y es acumulativo. Su gestión refuerza la sostenibilidad de las ventajas competitivas).

\section{REFERENCIAS BIBLIOGRÁFICAS}

1. Amit, R. Schoemaker, P. J. H. (1993). "Strategic assets and organizational rent". Strategic Management Journal, No 14: 33-46.

2. Barney, J. (1991). "Firms resources and Sustained competitive advantage". Journal of Management No 17: 99-120.

3. Camisón, C. (1997). La competitividad de la PYME industrial española: Estrategia $y$ competencias distintivas. Madrid: Editorial Civitas.

4. Fundación BBVA (2008). "Competitividad y crecimiento: una perspectiva regional". Cuadernos de trabajo N.o 8.

5. Grant, R.M. (1991). "The resource based theory of competitive advantage: implications for strategy formulation". California Management Review No 33: 114-135.

6. Hamel, G. Prahalad, C.K. (1999). Compitiendo por el futuro. Barcelona: Ariel Sociedad Económica.

7. Krugman, P. (1994). "Competitiveness: a dangerous obsession", Foreign Affairs No 73 (2): 28.

8. Porter, M. (1987). Ventaja competitiva. México: CEPSA.

9. Rumelt, R.P. (1974). Strategy, structure and economic performance. Cambrigde: Harvard University Press. 